\title{
STONE DISEASE
}

\section{Secondary signs of non-enhanced CT prior to laser ureterolithotripsy: is treatment outcome predictable?}

Seitz C, Memarsadeghi M, Fajkovic H, Tanovic E

Rohrbacher Str. 8, Medical University of Vienna, Austria

J Endourol. 2008; 22: 415-8

Purpose: To correlate the presence of secondary signs of non-enhanced computed tomography (NECT) in renal units harboring ureteral calculi with intraoperative findings and treatment outcome after holmium:yttrium-aluminum-garnet laser (Ho:YAG) ureterolithotripsy.

Subjects and Methods: Two-hundred patients were prospectively included after ureteral calculi were detected on NECT. All patients underwent Ho:YAG ureterolithotripsy at the Medical University of Vienna. All CT studies were reviewed by one specialized uroradiologist blinded to pre- and postoperative parameters for secondary signs as renal enlargement, perinephric stranding, ureteral dilation, periureteral edema, and ureteral rim sign. The impact of secondary signs on intraoperatively-verified impaction and treatment outcome was evaluated. Results: Of the 200 patients 85 (42.5\%) harbored proximal and 115 (57.5\%) harbored distal ureteral calculi. The stone-free rates for proximal and distal calculi were $80 \%$ and $97 \%$, respectively. Although proximal stone location and intraoperatively-verified impaction correlated significantly with stone-free rates $(\mathrm{P}<0.0001, \mathrm{P}=$ $0.01)$, the presence of secondary signs could not predict intraoperatively-verified stone impaction or stone-free rates (renal enlargement: $\mathrm{P}=0.2, \mathrm{P}=0.5$; perinephric stranding: $\mathrm{P}=0.7, \mathrm{P}=0.5$; ureteral dilation: $\mathrm{P}=0.7, \mathrm{P}=$ 0.7; periureteral edema: $\mathrm{P}=0.8, \mathrm{P}=0.06$; ureteral rim sign: $\mathrm{P}=0.8, \mathrm{P}=0.3$ ).

Conclusion: Preoperative secondary signs seen on NECT in patients harboring ureteral calculi do not correlate with intraoperative findings of impaction, and do not predict treatment outcome after Ho:YAG ureterolithotripsy.

\section{Editorial Comment}

Previous studies have demonstrated that patients with secondary signs of obstruction on CT scan imaging are more likely to require surgical intervention. It would be helpful if CT scan findings could predict the success rate with ureteroscopic lithotripsy. As such, the success rates are high and complication rates uncommon with Holmium laser lithotripsy, therefore the likelihood of identifying preoperative prognostic factors is low.

The low level of success with proximal ureteral stones could be related to the reliance on semi-rigid ureteroscopy in this study - the addition of flexible ureteroscopes and stone retrieval devices may have helped improve success rates. As such, the impact of proximal ureteral stone location and endoscopic evidence of impaction may warrant further evaluation using these two modalities.

The authors report that over one-third of patients had stone impaction at the time of ureteroscopy, as defined by adherence to the ureteral wall necessitating detachment with the Holmium laser. In our experience, the risk of impaction appears related to the duration of symptoms and obstructions. Indeed, it is surprising that such a high rate of impaction was detected as the median time to intervention was only 2 days after imaging.

Dr. Manoj Monga

Professor, Department of Urology University of Minnesota

Edina, Minnesota, USA

E-mail:endourol@yahoo.com 
Quantitative assessment of citric acid in lemon juice, lime juice, and commercially-available fruit juice products

Penniston KL, Nakada SY, Holmes RP, Assimos DG

Department of Surgery, Division of Urology, University of Wisconsin School of Medicine and Public Health, Madison, Wisconsin, North Carolina

J Endourol. 2008; 22: 567-70

Background and Purpose: Knowledge of the citric acid content of beverages may be useful in nutrition therapy for calcium urolithiasis, especially among patients with hypocitraturia. Citrate is a naturally-occurring inhibitor of urinary crystallization; achieving therapeutic urinary citrate concentration is one clinical target in the medical management of calcium urolithiasis. When provided as fluids, beverages containing citric acid add to the total volume of urine, reducing its saturation of calcium and other crystals, and may enhance urinary citrate excretion. Information on the citric acid content of fruit juices and commercially-available formulations is not widely known. We evaluated the citric acid concentration of various fruit juices.

Materials and Methods: The citric acid content of 21 commercially-available juices and juice concentrates and the juice of three types of fruits was analyzed using ion chromatography.

Results: Lemon juice and lime juice are rich sources of citric acid, containing 1.44 and $1.38 \mathrm{~g} / \mathrm{oz}$, respectively. Lemon and lime juice concentrates contain 1.10 and $1.06 \mathrm{~g} / \mathrm{oz}$, respectively. The citric acid content of commercially available lemonade and other juice products varies widely, ranging from 0.03 to $0.22 \mathrm{~g} / \mathrm{oz}$.

Conclusions: Lemon and lime juice, both from the fresh fruit and from juice concentrates, provide more citric acid per liter than ready-to-consume grapefruit juice, ready-to-consume orange juice, and orange juice squeezed from the fruit. Ready-to-consume lemonade formulations and those requiring mixing with water contain $</=6$ times the citric acid, on an ounce-for-ounce basis, of lemon and lime juice.

\title{
Editorial Comment
}

Citrate is the most abundant urinary organic ion and a potent inhibitor of crystallization, nucleation and crystal growth and aggregation. It acts by binding free calcium, binding to the calcium oxalate crystal surface, and blocking crystal-epithelial cell interactions. It may also impact urinary $\mathrm{pH}$. This evaluation of the citric acid concentration of various fruit juices answers important questions that will help with the dietary counseling of our patients with stone disease.

As with most good studies, it also provokes further questions worthy of investigation. How does the bioavailability of the dietary citrate sources differ, and how does each source impact urinary citrate and $\mathrm{pH}$ ? Is there variability in citrate contents based on the climate or soil composition where the fruits were grown? Though citrate levels are lower for orange juice, potassium levels are higher - would the added alkali load with orange juice enhance urinary citrate excretion and offset its lower citrate level?

For now, it is important we emphasize for patients that fresh or concentrated lemon or lime appears to be their best shot at squeezing their risk of stone recurrence.

\author{
Dr. Manoj Monga \\ Professor, Department of Urology \\ University of Minnesota \\ Edina, Minnesota, USA \\ E-mail:endourol@yahoo.com
}

